\title{
COMPOUND POISSON APPROXIMATION FOR EXTREMES OF MOVING MINIMA IN ARRAYS OF INDEPENDENT RANDOM VARIABLES
}

Abstract. We present conditions sufficient for the weak convergence to a compound Poisson distribution of the distributions of the $k$ th order statistics for extremes of moving minima in arrays of independent random variables.

1. Introduction. Let $\left\{X_{n, i}: i=1, \ldots, n, n=1,2, \ldots\right\}$ be an array of independent random variables with a common distribution function $F_{n}$ for fixed $n$. We define

$$
V_{n, j}=\min _{j \leq i<j+m_{n}} X_{n, i}, \quad j=1, \ldots, n-m_{n}+1,
$$

where $m_{n}$ is a sequence of positive integers. The array $\left\{V_{n, j}: j=1, \ldots, n-\right.$ $\left.m_{n}+1, n=1,2, \ldots\right\}$ is stationary and $\left(m_{n}-1\right)$-dependent in each row. Denote by

$$
\begin{aligned}
\min \left(V_{n, j}: j=\right. & \left.1, \ldots, n-m_{n}+1\right)=M_{n, m_{n}}^{\left(n-m_{n}+1\right)} \leq M_{n, m_{n}}^{\left(n-m_{n}\right)} \\
& \leq \ldots \leq M_{n, m_{n}}^{(1)}=\max \left(V_{n, j}: j=1, \ldots, n-m_{n}+1\right)
\end{aligned}
$$

the order statistics of the sequence $V_{n, 1}, \ldots, V_{n, n-m_{n}+1}$. In [2] E. R. Canfield and W. P. McCormick have obtained a limit law for $M_{n, m_{n}}^{(1)}$. They showed that if

$$
\frac{m_{n}}{\ln n} \rightarrow d \geq 0 \quad \text { as } n \rightarrow \infty
$$

then

$$
P\left\{M_{n, m_{n}}^{(1)} \leq u_{n}\right\} \rightarrow e^{-\theta \lambda} \quad \text { as } n \rightarrow \infty
$$

1991 Mathematics Subject Classification: 60F, 60E.

Key words and phrases: compound Poisson distribution, order statistics, moving minima, consecutive- $m$-out-of- $n$ system. 
where $\theta=1-\exp (-1 / d)$, while $\lambda>0$ and the sequence $\left\{u_{n}: n=1,2, \ldots\right\}$ of real numbers are related by

$$
n P^{m_{n}}\left\{X_{n, 1}>u_{n}\right\}=\lambda .
$$

In this paper we extend (4) to the case of any $k$ th order statistic. The limit law will be represented in terms of a compound Poisson distribution. Our result is also a generalization of [4] where Zubkov's method (see [7]) was used to obtain weak convergence of the distributions of the $k$ th order statistics (2) to the Poisson law under the condition

$$
m_{n} / \ln n \rightarrow 0 \quad \text { as } n \rightarrow \infty \text {. }
$$

The proofs of the main result of this paper are based on Stein's method (see $[1])$.

The problems considered have a connection with reliability theory. The random variables $M_{n, m_{n}}^{(1)}$ can be interpreted as lifetimes of consecutive- $m$ out-of- $n$ systems. Such a system fails if and only if at least $m$ consecutive components out of $n$ linearly ordered components fail. Some examples of applications to telecommunication and oil pipelines modelling may be found in [3] and [5].

2. Definitions and preliminary results. We say that a discrete random variable $W$ has a compound Poisson distribution if

$$
M(t)=E \exp (-t W)=\exp \left(-\sum_{n=1}^{\infty} c_{n}\left(1-e^{-t n}\right)\right)
$$

for all $t>0$, where $c_{n} \geq 0, n=1,2, \ldots$, are such that $0<\sum_{n=1}^{\infty} c_{n}<\infty$. Note that the corresponding distribution function is

$$
G\left(x,\left\{c_{n}\right\}\right)=\sum_{s \leq x} p_{s}\left(\left\{c_{n}\right\}\right), \quad x \in \mathbb{R},
$$

where

(8) $p_{s}\left(\left\{c_{n}\right\}\right)$

$$
= \begin{cases}\exp \left(-\sum_{n=1}^{\infty} c_{n}\right), & s=0, \\ \exp \left(-\sum_{n=1}^{\infty} c_{n}\right) \sum_{\substack{k_{1}+2 k_{2}+\ldots+s k_{s}=s \\ k_{j} \geq 0, j=1, \ldots, s}} \frac{c_{1}^{k_{1}} c_{2}^{k_{2}} \ldots c_{s}^{k_{s}}}{k_{1} ! k_{2} ! \ldots k_{s} !}, & s=1,2, \ldots\end{cases}
$$

The total variation distance between two probability measures $F$ and $G$ is defined by

$$
d(F, G)=\sup _{E}|F(E)-G(E)|,
$$


where the supremum is taken over all measurable subsets $E$ of the real line. Denote by $L(X)$ the law of a random variable $X$ and recall (see [6]) that if $d\left(L\left(X_{n}\right), L(X)\right) \rightarrow 0$ as $n \rightarrow \infty$ then $X_{n} \stackrel{w}{\rightarrow} X$ (weak convergence; see [6]). The following lemma will be used in the next section.

LEMma 1. Let $\left\{X_{n, i}: i=1, \ldots, n, n=1,2, \ldots\right\}$ be an array of independent random variables with a common distribution function $F_{n}$ for fixed $n$. If the sequence $\left\{m_{n}: n=1,2, \ldots\right\}$ of positive integers is such that

$$
\lim _{n \rightarrow \infty} m_{n} / \ln n=d, \quad d \geq 0,
$$

and

$$
\lim _{n \rightarrow \infty} n\left[1-F_{n}\left(u_{n}\right)\right]^{m_{n}}=\lambda
$$

where $\left\{u_{n}\right\}$ is a sequence of real numbers, then

$$
\lim _{n \rightarrow \infty} F_{n}\left(u_{n}\right)=1-e^{-1 / d} .
$$

Pr o of. From (10) we obtain

$$
\lim _{n \rightarrow \infty} \ln \left[n\left(1-F_{n}\left(u_{n}\right)\right)^{m_{n}}\right]=\ln \lambda
$$

Since

$$
\lim _{n \rightarrow \infty} \frac{\ln n+m_{n} \ln \left[1-F_{n}\left(u_{n}\right)\right]}{m_{n}}=\lim _{n \rightarrow \infty} \frac{\ln \lambda}{m_{n}}=0,
$$

we conclude from (9) that

$$
\lim _{n \rightarrow \infty}\left[1-F_{n}\left(u_{n}\right)\right]=e^{-1 / d} .
$$

3. The main results. Let $\left\{X_{n, i}: i=1, \ldots, n, n=1,2, \ldots\right\}$ be an array of independent random variables with a common distribution function $F_{n}$ for fixed $n$, and let $\left\{V_{n, j}: j=1, \ldots, n-m_{n}+1, n=1,2, \ldots\right\}$ be defined by (1). Consider an array $\left\{I_{n, j}: j=1, \ldots, n-m_{n}+1, n=1,2, \ldots\right\}$ of zero-one random variables $I_{n, j}=I_{\left\{V_{n, j}>u_{n}\right\}}$, where $u_{n}$ is a sequence of real numbers and $I_{A}$ denotes the indicator function of the set $A$. This last array is stationary and $\left(m_{n}-1\right)$-dependent in each row and

$$
\begin{aligned}
P\left\{I_{n, j}=1\right\} & =P\left\{I_{n, 1}=1\right\}=P\left\{V_{n, 1}>u_{n}\right\} \\
& =P\left\{X_{n, 1}>u_{n}, X_{n, 2}>u_{n}, \ldots, X_{n, m_{n}}>u_{n}\right\} \\
& =\left[1-F_{n}\left(u_{n}\right)\right]^{m_{n}} .
\end{aligned}
$$

Let us observe that $(m-1)$-dependence is a special case of local dependence defined in [1] with

$$
\begin{aligned}
& A_{\alpha}=\{\beta \in I:|\alpha-\beta|<m\}, \\
& B_{\alpha}=\{\beta \in I:|\alpha-\beta| \leq 2(m-1)\}, \quad I=\{1, \ldots, n\} .
\end{aligned}
$$


Set

We define, as in [1],

$$
S_{n}=\sum_{i=1}^{n-m_{n}+1} I_{n, i}
$$

$$
Y_{n, \alpha}=\sum_{\substack{|\alpha-\beta|<m_{n} \\ \alpha \neq \beta}} I_{n, \beta}, \quad \alpha=1, \ldots, n-m_{n}+1,
$$

and

$$
\lambda_{n, i}=\frac{1}{i} \sum_{\alpha=1}^{n-m_{n}+1} P\left\{I_{n, \alpha}=1, Y_{n, \alpha}=i-1\right\}, \quad i=1, \ldots, 2 m_{n}-1 .
$$

Let $M_{n, m_{n}}^{\left(n-m_{n}+1\right)} \leq \ldots \leq M_{n, m_{n}}^{(1)}$ be the order statistics of the sequence $V_{n, 1}, \ldots, V_{n, n-m_{n}+1}$ defined by $(2)$.

LEMma 2. For $k=1,2, \ldots$,

$$
\begin{aligned}
\mid P\left\{M_{n, m_{n}}^{(k)}\right. & \left.\leq u_{n}\right\}-G\left(k-1,\left\{\lambda_{n, i}\right\}\right) \mid \\
& \leq 2\left(1 \wedge \lambda_{n, 1}^{-1}\right) \exp \left(-\sum_{i=1}^{\infty} \lambda_{n, i}\right) \sum_{\alpha=1}^{n-m_{n}+1} \sum_{\beta \in B_{n, \alpha}} P_{n, \alpha} P_{n, \beta},
\end{aligned}
$$

where

$$
\begin{aligned}
& a \wedge b=\min (a, b) \\
& P_{n, \alpha}=P\left\{I_{n, \alpha}=1\right\}=\left[1-F_{n}\left(u_{n}\right)\right]^{m_{n}}, \\
& B_{n, \alpha}=\left\{\beta \in\left\{1, \ldots, n-m_{n}+1\right\}:|\alpha-\beta| \leq 2\left(m_{n}-1\right)\right\} .
\end{aligned}
$$

Proof. This follows from the equality $P\left\{M_{n, m_{n}}^{(k)} \leq u_{n}\right\}=P\left\{S_{n}<k\right\}=$ $P\left\{S_{n} \leq k-1\right\}$ and Theorem 8 of [1].

LEMMA 3. If

$$
\lim _{n \rightarrow \infty} n\left[1-F_{n}\left(u_{n}\right)\right]^{m_{n}}=\lambda, \quad \lambda>0,
$$

and

$$
\lim _{n \rightarrow \infty} m_{n} / \ln n=d \geq 0
$$

then

$$
\lim _{n \rightarrow \infty} \lambda_{n, i}=\lambda_{i}, \quad i=1,2, \ldots,
$$

where

$$
\lambda_{1}=\lambda, \quad \lambda_{i}=0, \quad i=2,3, \ldots, \quad \text { for } d=0,
$$

and

$$
\lambda_{i}=\lambda \theta^{2} e^{-(i-1) / d}, \quad i=1,2, \ldots, \quad \text { for } d>0 .
$$


Proof. We fix $i, i=1, \ldots, 2 m_{n}-1$. For each $n$ we divide the integers $1, \ldots, n-m_{n}+1$ in three parts:

$$
\begin{aligned}
& J_{n, 1}=\left\{1, \ldots, m_{n}-1\right\}, \\
& J_{n, 2}=\left\{m_{n}, \ldots, n-2 m_{n}+2\right\}, \\
& J_{n, 3}=\left\{n-2 m_{n}+3, \ldots, n-m_{n}+1\right\} .
\end{aligned}
$$

Because the array $\left\{I_{n, j}\right\}$ is stationary, we have

$$
\begin{aligned}
\lambda_{n, i}= & \frac{1}{i}\left(\sum_{\alpha=1}^{m_{n}-1} P\left\{I_{n, \alpha}=1, Y_{n, \alpha}=i-1\right\}\right. \\
& +\left(n-3 m_{n}+3\right) \sum_{j=0}^{i-1} P\left\{\sum_{k=1}^{m_{n}-1} I_{n, k}=j, I_{n, m_{n}}=1,\right. \\
& \left.+\sum_{\alpha=m_{n}+1}^{2 m_{n}-1} I_{n, k}=i-1-j\right\} \\
& \left.\sum_{\alpha=2 m_{n}+3}^{n-m_{n}+1} P\left\{I_{n, \alpha}=1, Y_{n, \alpha}=i-1\right\}\right) .
\end{aligned}
$$

Define

$$
\begin{aligned}
& R_{n, j}=\left\{\sum_{k=1}^{m_{n}-1} I_{n, k}=j, I_{n, m_{n}}=1, \sum_{k=m_{n}+1}^{2 m_{n}-1} I_{n, k}=i-1-j\right\} \\
& j=0, \ldots, i-1 .
\end{aligned}
$$

Observe that events of the form $\left\{\ldots, V_{n, i}>u_{n}, V_{n, i+1} \leq u_{n}, V_{n, i+2}>\right.$ $\left.u_{n}, \ldots\right\}$ are impossible because $\left\{X_{n, i+m_{n}} \leq u_{n}\right\}$ and $\left\{X_{n, i+m_{n}}>u_{n}\right\}$ are mutually exclusive. Thus

$$
\begin{aligned}
R_{n, j}=\left\{V_{n, 1} \leq\right. & u_{n}, \ldots, V_{n, m_{n}-j-1} \leq u_{n}, \\
& V_{n, m_{n}-j}>u_{n}, \ldots, V_{n, m_{n}}>u_{n}, \ldots, V_{n, m_{n}+i-j-1}>u_{n}, \\
& \left.V_{n, m_{n}+i-j} \leq u_{n}, \ldots, V_{n, 2 m_{n}-1} \leq u_{n}\right\} .
\end{aligned}
$$

We fix $j=0, \ldots, i-1$. By the definition of $\left\{I_{n, j}\right\}$ and $\left\{V_{n, j}\right\}$, and the assumptions on $\left\{X_{n, i}\right\}$, we have

$$
P\left\{R_{n, j}\right\}=\sum_{l=0}^{m_{n}-j-2} \sum_{p=0}^{m_{n}-i+j-1} P\left\{\sum_{k=1}^{m_{n}-j-2} I_{\left\{X_{n, k}>u_{n}\right\}}=l,\right.
$$




$$
\begin{aligned}
& \sum_{k=m_{n}-j}^{2 m_{n}+i-j-2} I_{\left\{X_{n, k}>u_{n}\right\}}=m_{n}+i-1, I_{\left\{X_{n, 2 m_{n}+i-j-1}>u_{n}\right\}}=0, \\
& \left.\sum_{k=2 m_{n}+i-j}^{3 m_{n}-2} I_{\left\{X_{n, k}>u_{n}\right\}}=p\right\} \\
& =\left[1-F_{n}\left(u_{n}\right)\right]^{m_{n}+i-1} F_{n}^{2}\left(u_{n}\right) .
\end{aligned}
$$

Because $P\left\{R_{n, j}\right\}$ does not depend on $j$, for each $0 \leq j, k \leq i-1$ we have

$$
P\left\{R_{n, j}\right\}=P\left\{R_{n, k}\right\} .
$$

Next, set

$$
K_{n}(i)=\left(n-3 m_{n}+3\right) \sum_{j=0}^{i-1} P\left\{R_{n, j}\right\} .
$$

From (16) we obtain

$$
\begin{aligned}
K_{n}(i) & =i\left(n-3 m_{n}+3\right) P\left\{R_{n, 0}\right\} \\
& =i\left(n-3 m_{n}+3\right)\left[1-F_{n}\left(u_{n}\right)\right]^{m_{n}+i-1} F_{n}^{2}\left(u_{n}\right) .
\end{aligned}
$$

Hence

$$
\begin{aligned}
\frac{1}{i} K_{n}(i)= & \left(n-3 m_{n}+3\right)\left[1-F_{n}\left(u_{n}\right)\right]^{m_{n}} \\
& \times F_{n}^{2}\left(u_{n}\right)\left[1-F_{n}\left(u_{n}\right)\right]^{i-1} .
\end{aligned}
$$

Using Lemma 1 and (14) we have the following result: for $d=0$,

$$
\lim _{n \rightarrow \infty} \frac{1}{i} K_{n}(i)= \begin{cases}0, & i>1, \\ \lambda, & i=1,\end{cases}
$$

and

$$
\lim _{n \rightarrow \infty} \frac{1}{i} K_{n}(i)=\lambda \theta^{2} e^{-(i-1) / d} \quad \text { for } d>0 .
$$

Now let

$$
\begin{aligned}
& L_{n}^{(1)}(i)=\sum_{\alpha=1}^{m_{n}-1} P\left\{I_{n, \alpha}=1, Y_{n, \alpha}=i-1\right\}, \\
& L_{n}^{(2)}(i)=\sum_{\alpha=n-2 m_{n}+3}^{n-m_{n}+1} P\left\{I_{n, \alpha}=1, Y_{n, \alpha}=i-1\right\} .
\end{aligned}
$$

Our purpose is to show that

$$
\lim _{n \rightarrow \infty} L_{n}^{(r)}(i)=0, \quad r=1,2 .
$$


Set

$$
\begin{aligned}
& A_{n, \alpha}(j)=\left\{\sum_{k=1}^{\alpha-1} I_{n, k}=j, I_{n, \alpha}=1, \sum_{k=\alpha+1}^{\alpha+m_{n}-1} I_{n, k}=i-j-1\right\}, \\
& \alpha=1, \ldots, m_{n}-1,0 \leq j \leq i-1 .
\end{aligned}
$$

Then

$$
L_{n}^{(1)}(i)=\sum_{\alpha<i} \sum_{j=0}^{i-1} P\left\{A_{n, \alpha}(j)\right\}+\sum_{i \leq \alpha \leq m_{n}} \sum_{j=0}^{i-1} P\left\{A_{n, \alpha}(j)\right\} .
$$

Note that

$$
\begin{aligned}
& A_{n, 1}(0)=\left\{V_{n, 1}>u_{n}, \ldots, V_{n, i}>u_{n}, V_{n, i+1} \leq u_{n}, \ldots, V_{n, m_{n}} \leq u_{n}\right\}, \\
& A_{n, \alpha}(j)=\left\{V_{n, 1} \leq u_{n}, \ldots, V_{n, \alpha-j-1} \leq u_{n}\right. \text {, } \\
& V_{n, \alpha-j}>u_{n}, \ldots, V_{n, \alpha}>u_{n}, \ldots, V_{n, \alpha+i-1-j}>u_{n}, \\
& \left.V_{n, \alpha+i-j} \leq u_{n}, \ldots, V_{n, \alpha+m_{n}-1} \leq u_{n}\right\} \text {. }
\end{aligned}
$$

Now it is easy to see that $A_{n, \alpha}(j) \subset C_{n, \alpha}(j)$ where

$$
\begin{array}{r}
C_{n, \alpha}(j)=\left\{V_{n, \alpha-j}>u_{n}, \ldots, V_{n, \alpha}>u_{n}, \ldots, V_{n, \alpha+i-1-j}>u_{n},\right. \\
\left.V_{n, \alpha+i-j} \leq u_{n}, \ldots, V_{n, \alpha+m_{n}-1} \leq u_{n}\right\} .
\end{array}
$$

From the stationarity of the array $\left\{V_{n, j}\right\}$,

$$
P\left\{C_{n, \alpha}(j)\right\}=P\left\{D_{n, \alpha}(j)\right\},
$$

where

$$
\begin{aligned}
D_{n, \alpha}(j)=\left\{V_{n, 1}>u_{n}, \ldots, V_{n, j+1}>\right. & u_{n}, \ldots, V_{n, i}>u_{n}, \\
& \left.V_{n, i+1} \leq u_{n}, \ldots, V_{n, m_{n}+j} \leq u_{n}\right\} .
\end{aligned}
$$

It is obvious that $D_{n, \alpha}(j) \subset A_{n, 1}(0)$ so

$$
P\left\{A_{n, \alpha}(j)\right\} \leq P\left\{A_{n, 1}(0)\right\}
$$

for $j=0, \ldots, \alpha-1$ if $\alpha<i$, and for $j=0, \ldots, i-1$ otherwise. From (18) and the assumed properties of $\left\{X_{n, j}\right\}$ and $\left\{V_{n, j}\right\}$ we obtain

$$
\begin{aligned}
L_{n}^{(1)}(i) \leq & \left(m_{n}-1\right) i\left[1-F_{n}\left(u_{n}\right)\right]^{m_{n}+i-1} F_{n}\left(u_{n}\right) \\
& \times \sum_{s=0}^{m_{n}-i-1}\left(\begin{array}{c}
m_{n}-i-1 \\
s
\end{array}\right)\left[1-F_{n}\left(u_{n}\right)\right]^{s} F_{n}^{m_{n}-i-1-s}\left(u_{n}\right) \\
= & \frac{m_{n}-1}{n} \cdot i \cdot n\left[1-F_{n}\left(u_{n}\right)\right]^{m_{n}} F_{n}\left(u_{n}\right)\left[1-F_{n}\left(u_{n}\right)\right]^{i-1} .
\end{aligned}
$$

Note that in view of $(15), m_{n}=o(n)$, which together with the assumption (14) and Lemma 1 implies that the right-hand side of (19) tends to zero as $n \rightarrow \infty$. The same is true for $L_{n}^{(2)}(i)$. 
Finally, for $d=0$,

and for $d>0$,

$$
\lim _{n \rightarrow \infty} \lambda_{n, i}= \begin{cases}\lambda, & i=1, \\ 0, & i=2, \ldots, 2 m_{n}-1,\end{cases}
$$

$$
\lim _{n \rightarrow \infty} \lambda_{n, i}=\lambda \theta^{2} e^{-(i-1) / d}, \quad i=1, \ldots, 2 m_{n}-1 .
$$

LEMMA 4. We have

$$
\lim _{n \rightarrow \infty} \sum_{i=1}^{\infty} \lambda_{n, i}=\sum_{i=1}^{\infty} \lambda_{i}=\lambda \theta .
$$

Proof. The second equality of (20) follows simply, since

$$
\sum_{i=1}^{\infty} \lambda_{i}=\lambda \theta^{2} \frac{1}{1-e^{-1 / d}}=\lambda \theta
$$

Next, for fixed $n$, because of (17) and (19), we obtain

$$
\lambda_{n, i} \leq n\left[1-F_{n}\left(u_{n}\right)\right]^{m_{n}} F_{n}\left(u_{n}\right)\left[1-F_{n}\left(u_{n}\right)\right]^{i-1} .
$$

Hence, from Lemma 1,

$$
\lambda_{n, i} \leq \lambda\left(1-e^{-1 / d}\right) e^{-(i-1) / d} .
$$

Set

and note that

$$
a_{i}=\lambda \theta e^{-(i-1) / d}
$$

$$
\sum_{i=1}^{\infty} a_{i}=\lambda \theta \frac{1}{1-e^{-1 / d}}=\lambda<\infty .
$$

Hence the series $\sum_{i=1}^{\infty} \lambda_{n, i}$ is uniformly convergent and thus in view of Lemma 3 we have $(20)$.

LEMMA 5. If (14) and (15) hold, then

$$
\lim _{n \rightarrow \infty} \sum_{\alpha=1}^{n-m_{n}+1} \sum_{\beta \in B_{n, \alpha}} P_{n, \alpha} P_{n, \beta}=0,
$$

where $P_{n, \alpha}$ and $B_{n, \alpha}$ are as in Lemma 2 .

Proof. Since $P_{n, \alpha}=\left[1-F_{n}\left(u_{n}\right)\right]^{m_{n}}$, we have

$$
\begin{aligned}
\sum_{\alpha=1}^{n-m_{n}+1} \sum_{\beta \in B_{n, \alpha}} P_{n, \alpha} P_{n, \beta} & \leq 2\left(n-m_{n}+1\right)\left(m_{n}-1\right)\left[1-F_{n}\left(u_{n}\right)\right]^{2 m_{n}} \\
& =2 \frac{n-m_{n}+1}{n} \cdot \frac{m_{n}-1}{n} n^{2}\left[1-F_{n}\left(u_{n}\right)\right]^{2 m_{n}} .
\end{aligned}
$$

The right side converges to zero as $n \rightarrow \infty$ by (14) and (15). 
The main result of this paper may now be readily proved.

THEOREM 1. Let $\left\{m_{n}\right\}$ be a sequence of positive integers satisfying (15) and $\left\{u_{n}\right\}$ a sequence of real numbers satisfying (14). Then for $k=1,2, \ldots$,

$$
\lim _{n \rightarrow \infty} P\left\{M_{n, m_{n}}^{(k)} \leq u_{n}\right\}=G\left(k-1,\left\{\lambda_{i}\right\}\right)
$$

where the distribution function $G$ is given by $(7)-(8)$.

Proof. We have

$$
\begin{aligned}
\mid P\left\{M_{n, m_{n}}^{(k)} \leq\right. & \left.u_{n}\right\}-G\left(k-1,\left\{\lambda_{i}\right\}\right) \mid \\
\leq & \left|P\left\{M_{n, m_{n}}^{(k)} \leq u_{n}\right\}-G\left(k-1,\left\{\lambda_{n, i}\right\}\right)\right| \\
& +\left|G\left(k-1,\left\{\lambda_{n, i}\right\}\right)-G\left(k-1,\left\{\lambda_{i}\right\}\right)\right|, \quad k=1,2, \ldots
\end{aligned}
$$

From Lemma 2,

$$
\begin{aligned}
\mid P\left\{M_{n, m_{n}}^{(k)}\right. & \left.\leq u_{n}\right\}-G\left(k-1,\left\{\lambda_{n, i}\right\}\right) \mid \\
& \leq 2\left(1 \wedge \lambda_{n, 1}^{-1}\right) \exp \left(-\sum_{i=1}^{\infty} \lambda_{n, i}\right) \sum_{\alpha=1}^{n-m_{n}+1} \sum_{\beta \in B_{n, \alpha}} P_{n, \alpha} P_{n, \beta} .
\end{aligned}
$$

Note that $\lim _{n \rightarrow \infty} \lambda_{n, 1}=\lambda \theta^{2}$ and since $\lim _{n \rightarrow \infty} \sum_{i=1}^{\infty} \lambda_{n, i}=\lambda \theta$ we have

$$
\lim _{n \rightarrow \infty} \exp \left(-\sum_{i=1}^{\infty} \lambda_{n, i}\right)=\exp (-\lambda \theta) .
$$

Thus from (21) the right side of (24) tends to zero as $n \rightarrow \infty$. For $k=1,2, \ldots$ we also have

$$
\begin{gathered}
\left|G\left(k-1,\left\{\lambda_{n, i}\right\}\right)-G\left(k-1,\left\{\lambda_{i}\right\}\right)\right| \\
\leq \sum_{s<k} \mid \exp \left(-\sum_{i=1}^{\infty} \lambda_{n, i}\right) \sum_{\substack{k_{1}+2 k_{2}+\ldots+s k_{s}=s \\
k_{j} \geq 0, j=1, \ldots, s}} \frac{\lambda_{n, 1}^{k_{1}} \lambda_{n, 2}^{k_{2}} \ldots \lambda_{n, s}^{k_{s}}}{k_{1} ! k_{2} ! \ldots k_{s} !} \\
-\exp (-\lambda \theta) \sum_{\substack{k_{1}+2 k_{2}+\ldots+s k_{s}=s \\
k_{j} \geq 0, j=1, \ldots, s}} \frac{\lambda_{1}^{k_{1}} \lambda_{2}^{k_{2}} \ldots \lambda_{s}^{k_{s}}}{k_{1} ! k_{2} ! \ldots k_{s} !} \mid .
\end{gathered}
$$

Note that for fixed $k$ we have a finite number of terms in the last two sums. Hence by (25) and Lemma 3 the right side of the inequality (23) converges to zero as $n \rightarrow \infty$.

As an immediate corollary of Theorem 1 we easily obtain the result of Canfield and McCormick [2]. 
COROLlary 1. Let $\left\{m_{n}\right\}$ and $\left\{u_{n}\right\}$ be sequences satisfying (15) and (14) respectively. Then

$$
\lim _{n \rightarrow \infty} P\left\{M_{n, m_{n}}^{(1)} \leq u_{n}\right\}=e^{-\lambda \theta} .
$$

P r oof. Using Theorem 1 for $k=1$ we obtain

$$
\lim _{n \rightarrow \infty} P\left\{M_{n, m_{n}}^{(1)} \leq u_{n}\right\}=G\left(0,\left\{\lambda_{i}\right\}\right)
$$

where

$$
G\left(0,\left\{\lambda_{i}\right\}\right)=p_{0}\left(\left\{\lambda_{i}\right\}\right)=\exp \left(-\sum_{i=1}^{\infty} \lambda_{i}\right)=\exp (-\lambda \theta)
$$

\section{References}

[1] A. D. Barbour, L. H. Y. Chen and W. L. Loh, Compound Poisson approximation for nonnegative random variables via Stein's method, Ann. Probab. 20 (1992), 18431866.

[2] E. R. Canfield and W. P. McCormick, Asymptotic reliability of consecutive $k$ out-of-n systems, J. Appl. Probab. 29 (1992), 142-155.

[3] O. Chryssaphinou and S. G. Papastavridis, Limit distribution for a consecutive k-out-of-n:F system, Adv. Appl. Probab. 22 (1990), 491-493.

[4] J. Dudkiewicz, Asymptotic of extremes of moving minima in arrays of independent random variables, Demonstratio Math. 29 (1996), 715-721.

[5] S. G. Papastavridis, A limit theorem for the reliability of a consecutive-k-out-of-n system, Adv. Appl. Probab. 19 (1987), 746-748.

6] R. J. Serfling, A general Poisson approximation theorem, Ann. Probab. 3 (1975), $726-731$.

[7] A. M. Zubkov, Estimates for sums of finitely dependent indicators and for the time of first occurrence of a rare event, Probabilistic Problems of Discrete Mathematics, Trudy Mat. Inst. Steklov. 177 (1986), 33-46, 207 (in Russian).

Jadwiga Dudkiewicz

Institute of Mathematics

Technical University of Kielce

Tysiaclecia PP 7

25-314 Kielce, Poland 